\title{
Liquid-Liquid Phase Separation inside Giant Vesicles Drives Shape Deformations and Induces Lipid Membrane Phase Separation
}

\section{Wan-Chih Su}

University of California, Davis

\section{Douglas Gettel}

University of California, Davis

\section{Andrew Rowland}

The Pennsylvania State University

\section{Christine Keating}

Pennsylvania State University https://orcid.org/0000-0001-6039-1961

Atul Parikh ( $\square$ anparikh@ucdavis.edu )

University of California, Davis https://orcid.org/0000-0002-5927-4968

\section{Article}

Keywords: liquid-liquid phase separation, kinetic pathways, thermodynamics

Posted Date: September 7th, 2021

DOI: https://doi.org/10.21203/rs.3.rs-827501/v1

License: (c) (1) This work is licensed under a Creative Commons Attribution 4.0 International License. Read Full License 


\section{Liquid-Liquid Phase Separation inside Giant Vesicles Drives Shape Deformations and Induces}

Lipid Membrane Phase Separation

3

\author{
Wan-Chih Su${ }^{1}$, Doug L. Gettel ${ }^{2}$, Andrew T. Rowland ${ }^{3}$, Christine D. Keating*,3, Atul N. Parikh*1,2,4 \\ ${ }^{1}$ Chemistry, ${ }^{2}$ Chemical Engineering, and ${ }^{4}$ Biomedical Engineering Graduate Programs, University of \\ California, Davis, California 95616, USA \\ ${ }^{3}$ Department of Chemistry, The Pennsylvania State University, University Park, PA 16802, USA
}

An astounding variety of cellular contexts converge to the process of liquid-liquid phase separation for the creation of new functional levels of organization. But the kinetic pathways by which intracellular phase separation proceeds - typically in physically confined and macromolecularly crowded volumes of topologically closed cellular and intracellular compartments -remain incompletely understood. Here, we monitor the dynamics of liquid-liquid phase separation of mixtures of phase-separating polymers (i.e., polyethyleneglycol and dextran) inside all-synthetic, cell-sized giant unilamellar vesicles in real-time. We dynamically trigger phase separation by subjecting an initially homogeneous polymer solution inside vesicles to an abrupt osmotic quench. The latter removes water and elevates polymer concentrations in the phase-coexistence regime thereby initiating a segregative phase separation of the polymers. We find that the ensuing relaxation - en route to the new equilibrium - is non-trivially modulated by a dynamic interplay between the coarsening of the evolving droplet phase and the interactive membrane boundary. The early trajectory of droplet coarsening exhibit significant acceleration, but a competing process of membranedroplet interactions - one in which the membrane boundary is preferentially wetted by one of the incipient phases - dynamically arrests the progression and deforms the membrane. As a result, a novel multi-bud morphology, reminiscent of cellular blebs, decorate the vesicle surface. Furthermore, when the vesicles are composed of phase-separating mixtures of common lipids, the three-dimensional liquid-liquid phase separation within the vesicular interior becomes coupled to the membrane's compositional degrees of freedom producing microphase-separated membrane textures. This coupling of bulk and surface phase separation processes suggests a new physical principle by which liquid-liquid phase separation inside living cells might be dynamically regulated and materially communicated inside-out to the cellular boundaries. 


\section{[MAIN TEXT]}

When a binary fluid mixture is quenched from a uniform homogeneous phase into the co-existence region, it enters into a far from equilibrium state $(1,2)$. The subsequent evolution of this non-equilibrium system in which the disordered "mother" phase gives way to new co-existing phases at equilibrium - is not instantaneous: Instead, complementary regions of the new broken-symmetry phases grow and coarsen over time. Here, growth corresponds to an increase in the volume fraction of the droplet phase via the transport of molecules from the complementary matrix phase. Coarsening, which follows growth, represents the morphological evolution due to the changes in the droplet size at the fixed, equilibrated value of the volume fraction( $(\underline{3})$. In both cases, the kinetic pathways of phase separation are determined by material transport via a combination of diffusion- and flow-mediated mechanisms, ultimately producing phase-separated morphologies of droplets enriched in either component. This general phenomenology of phase-ordering governs the evolution of compositional patterns during phase separation dictating equilibrium textures of phase-separated systems across many areas of materials science, including metal alloys, magnetic solids, polymers, colloids, and liquid-crystals( $\underline{3})$.

But all material systems undergo phase separation within finite containers. The physical limits the container boundaries impose - including finite system size and the proximity of the often selectively wetting bounding surface - can influence phase separation, perturb diffusive and hydrodynamic processes, and alter morphological evolution(4). Consider, for example, a surface, which preferentially attracts or wets one of the incipient co-existing phases over the other. As a consequence, it may spatially pattern the two phases altering the course and the kinetics of phase separation, thereby influencing the morphological evolution( 5 , 6). This phenomenon of surface-directed spinodal decomposition is perhaps best exemplified by studies of polymer mixtures undergoing liquid-liquid phase separation. These studies document a rich variety of ways by which solid surfaces intervene incipient phase separation. Examples include formation of compositional waves in the vicinity of the surface (ㄱ), acceleration of domain coarsening, (ㅁ, $\underline{9})$, and emergence of novel types of double phase separation( $\underline{10})$.

These behaviors are of particular interest in soft matter and biological systems - such as aqueous solutions of proteins and polymers in biological (i.e., cellular and sub-cellular) and synthetic (i.e., vesicular) compartments (11) - undergoing liquid-liquid phase separation (LLPS) (12). Here, phase separation proceeds in physically confined (femto- to picoliter quantities) and macromolecularly crowded volumes within topologically closed compartments. Moreover, the compartmental boundaries are neither passive nor irresponsive; they are fluid, deformable, and molecularly heterogeneous capable of interacting 
differentially with the incipient phases. This variety of system attributes - namely surface wetting, spatial confinement, surface deformability, and membrane's compositional degrees of freedom - can be expected to influence LLPS in complex ways, thus setting a stage for significantly richer and dramatically more complex phase behaviors in confined soft matter and biological systems in comparison with their counterparts in traditional materials science.

In considering real-time phase separation under the global constraints of vesicular confinement, several general questions naturally arise: (1) Are growth and phase coarsening limited to the intravesicular bulk or may surface direct LLPS? How are the kinetics influenced by the system size, geometry, and the proximity of the membrane surface? (2) Might the preferential wetting by the droplets deform the membrane and induce localized membrane remodeling? Does it spatially reorganize membrane components, possibly coupling LLPS within the vesicular bulk (3D) with the lipid-lipid phase separation (2D) within the membrane? (3) Does the interplay between phase separation and wetting arrest the relaxation of the coarsening system into a metastable minimum instead of the true thermodynamic ground state? Addressing these questions is particularly timely (11) given the rapidly growing recognition of the prevalence of LLPS across biology and its purported role as a driver for the formation of biomolecular condensates - a critical level of functional organization within a living cell( $\underline{13-18})$. Indeed, a growing body of recent observations suggest that there exists a complex interplay between intracellular LLPS and membrane phase separation at cellular boundaries(19-21). Moreover, understanding the physical principles governing the progression of LLPS within vesicular confinements is critically important for reconstituting these essential cellular mechanisms in synthetic cell-like entities(22-25).

Motivated by these questions, we experimentally investigate here a model case of phase ordering inside single vesicular compartments in situ. Our experimental strategy is simple. The phase ordering system we employ is a minimal aqueous two-phase systems (ATPS) consisting of aqueous mixtures of two chemically dissimilar and electrically neutral polymers: polyethylene glycol (PEG) and dextran (26). First developed for biomolecular separations, the ATPS system of PEG and dextran is a non-associative or segregative kind(26). At low weight fractions, these mixtures adopt a single homogeneous phase. Beyond a threshold concentration, they phase-separate, producing two co-existing phases at equilibrium with each phase enriching a different polymer. A binodal line dividing the two regions characterize the phase diagram of

31 these mixtures (26). The thermodynamic driving forces for the phase separation include synergistic 32 contributions(27) from two distinct molecular mechanisms: (1) energetically unfavorable interactions 33 between the segments of the two polymers overcoming the loss of mixing entropy(28) and (2) the repulsive 34 force or an "epistructural tension" arising from the differences in water structure surrounding the two 
polymers(29). Because of the weak nature of these interactions, the effective interfacial tension between

2 the two aqueous phases is low- three to four orders of magnitude lower than typical liquid-liquid systems

3 (30) - and highly adjustable. Its magnitude ranges between 1-1000 $\mu \mathrm{N} \mathrm{m}^{-1}$ and depends sensitively on the

4 proximity to the critical point and polymer properties, including concentrations, molecular weights, and the

5 disparities in their molecular weights $(\underline{31}, \underline{32})$. Moreover, both PEG and dextran are also efficient crowding

6 agents capable of minimally recapitulating macromolecular crowding, such as exists in cellular

7 cytoplasm( $\underline{33})$.

9 Our vesicular compartments, which encapsulate ATPS components in an initially homogeneous phase, are 10 giant unilamellar vesicles (GUVs)( $\underline{34}, \underline{35}$ ). They are the simplest, cell-sized ( $r \cong 5-25 \mu \mathrm{m}$ in radius), 11 topologically closed, and nominally spherical synthetic compartments, which isolate pico-liter quantities of 12 the encapsulated aqueous phase from the surrounding bulk. The GUVs are bounded by a two-dimensionally 13 fluid, highly deformable, and water-permeable $\left(P_{\text {water }} \approx 10^{-3} \mathrm{~cm} \cdot \mathrm{s}^{-1}\right)$ but solute-impermeable $\left(P_{\text {solute }} \approx 10^{-}\right.$

$\left.14{ }^{8}-10^{-10} \mathrm{~cm} \cdot \mathrm{s}^{-1}\right)$ bilayer lipid membrane $(\underline{36}, \underline{37})$. To induce LLPS in real-time, we subject the GUVs to an 15 abrupt hypertonic stress, which by rapidly expelling water from the intravesicular lumen (characteristic 16 permeation time $(\underline{38}),{ }^{r} / 3 P_{\text {water }}=\left(10 \times 10^{-4}\right) /\left(3 \times 10^{-3}\right) \approx 0.3 \mathrm{~s}$, abruptly elevates the polymer 17 concentrations, and quenches the mixtures inside the co-existence curve (Fig. 1a). The methods of 18 encapsulation of ATPS components within vesicular compartments $(39, \underline{40})$ and their osmotically-mediated 19 phase separation $(\underline{32}, \underline{41}, \underline{42})$ are well-established in recent literature. The use of aqueous phase separation inside vesicular compartments was first developed $(39, \underline{40})$ to minimally recapitulate physical features of cytosolic micro-compartmentation and macromolecular crowding such as occurs in living cells( $\underline{43-45})$. Since these early demonstrations, many studies have explored the equilibrium behaviors of ATPSencapsulating GUVs. In a series of studies, Dimova, Lipowsky, and others have investigated how equilibrated droplets interact with the bounding membrane(46-49). These efforts establish the role of surface wetting, which, together with membrane mechanics, offers important insights and quantitative explanations of the features of equilibrium morphologies, namely droplet-induced budding and membrane tubulation arising from LLPS.

Here, we monitor the real-time the progression of liquid-liquid phase separation of mixtures of PEG and dextran inside giant unilamellar vesicles, which ensues upon an osmotically-mediated quench inside the co-existence region of the phase diagram. We find that the relaxation pathway en route to the new

32 equilibrium is non-trivially modulated by a dynamic interplay between the late-stage coarsening of the 33 evolving droplet phase and the interactive membrane boundary. First, the droplet composition and the 
dominant length scale - characterizing the initial growth and evolving morphology respectively - diverge with time in an unusual manner: the growth, reflecting compositional equilibration, is dramatically slowed and the droplet coarsening exhibits a non-trivial acceleration. Second, the droplet coarsening does not persist uninterrupted till complete phase separation. A competing process of preferential wetting of the membrane boundary by one of the incipient phases dynamically arrests coarsening and locally deforms the membrane boundary producing a characteristic multi-bud morphology, reminiscent of budding in dying apoptotic cells and asymmetric vesicle division. Third, in multi-component vesicles consisting of phaseseparating lipid mixtures, the three-dimensional LLPS within the vesicular bulk becomes coupled to membrane's compositional degrees of freedom inducing isothermal lipid-lipid phase transition and producing microphase-separated membrane textures. Collectively, these findings highlight some of the most significant physical-chemical consequences of surface interactions, which may explain how liquidliquid phase separation inside living cells might be regulated and communicated inside-out to the cellular boundaries.

We begin by preparing giant unilamellar vesicles (GUVs, 10-50 $\mu \mathrm{m}$ in diameter) using the standard electroformation technique (므). For single-component GUVs we use monounsaturated phospholipid, namely 1-palmitoyl 2-oleoyl-sn-1-glycero-3-phosphocholine (POPC) and our ternary GUVs include predetermined mixtures containing POPC, cholesterol (Ch), and sphingomyelin (SM). Depending on the composition, temperature, membrane tension, and solution conditions, the latter mixture forms a single uniform phase or exhibits microscopic phase separation with the latter characterized by two co-existing liquid phases: a dense phase enriched in SM and Ch designated as the $\mathrm{L}_{\mathrm{o}}$ (liquid-ordered) phase and a second, less dense $\mathrm{L}_{d}$ (liquid-disordered) phase consisting primarily of POPC (1ㅡ). In both cases, we introduce a small concentration of methoxy (polyethylene glycol) derivatized 1,2-dioleoyl-sn-glycero-3phosphatidylethanolamine (DOPE-mPEG 2000, 2.2 mol\%) or monosialoganglioside (GM1, 4 mol\%), which are known to facilitate reproducible formation of GUVs $(\underline{52}, \underline{53})$. To enable visualization of the membrane by fluorescence microscopy, the GUVs are spiked with a small concentration $(1.0 \mathrm{~mol} \%)$ of a probe lipid, namely 1,2-dioleoyl-sn-glycero-3-phosphoethanolamine-N-(lissamine rhodamine B sulfonyl) (Rho-DOPE). In selected experiments employing phase-separating lipid mixtures, we also used a second fluorescent probe, 1,2-dioleoyl-sn-glycero-3-phosphoethanolamine-N-(7-nitro-2-1,3-benzoxadiazol-4-yl)

30 (ammonium salt) (NBD-PE, $3 \mathrm{~mol} \%$ ), which exhibits a greater preference for partitioning into the liquid31 ordered phase(54). To encapsulate the ATPS inside GUVs, we use aqueous solutions containing mixtures 32 of PEG $\left(8 \mathrm{~kg} \mathrm{~mol}^{-1}\right)$ and dextran $\left(10 \mathrm{~kg} \mathrm{~mol}^{-1}\right)(43)$ during the hydration step of electroformation, which 33 are known to encapsulate at concentrations comparable to that in the surrounding bath $(\underline{32}, \underline{49}, \underline{53})$. In all 34 cases, we chose initial concentrations that exist as a single homogeneous phase. As a guide, we use the 
1 binodal phase diagram of their bulk solutions, which we determined using the standard cloud-point 2 determination ( $\underline{55}$ ) (Fig. 1b).

4 Substituting the extra-vesicular dispersion medium with an aqueous phase containing osmotically active 5 sucrose (100-300 $\mathrm{mM}$ ) produces a hypertonic bath, subjecting the GUV to a trans-bilayer osmotic gradient.

6 The accompanying osmotic efflux of water from the vesicular interior elevates the concentrations of PEG 7 and dextran polymers in the GUV interior above the binodal line triggering phase separation in situ (Fig. 8 1a). The new co-existing phases formed upon hyperosmotic stress differ in their composition and density 9 and thus viscosity and refractive index. Bulk assays confirm that the dextran-rich phase is denser and 10 displays a higher refractive index( $(\underline{26})$. Combining bulk polarimetric measurements with refractometry 11 measurements, allows for an initial assessment of phase compositions: For each of the two co-existing 12 phases, the former estimates dextran concentration from optical rotation data and the latter measures total 13 polymer concentration in each of the two phases. The two molecular compositions correspond to the end 14 points of the tie lines and quantify that the fractionation of the two polymers between the co-existing phases.

15 Our results summarized in Fig. 1c confirm efficient fractionation, especially at higher dextran concentrations: The PEG-rich phase contains vanishing concentrations of dextran and conversely the dextran-rich phase has only a small concentration of PEG. These observations also confirm that the total polymer loading in the dextran-rich phase is considerably higher than in the PEG-rich phase, consistent with the greater density of the dextran-rich phase.

Monitoring the dynamics of phase separation using spinning disc confocal fluorescence microscopy documents the phase transition trajectory. A selection of images from the time-lapse movie (Supplementary Video S1) is shown in Fig. 2a-c upon the immersion of single-component POPC GUVs formed in a solution containinf $6 \mathrm{wt} \% \operatorname{PEG}\left(8 \mathrm{~kg} \mathrm{~mol}^{-1}\right)$ and $6.4 \mathrm{wt} \% \operatorname{dextran}\left(10 \mathrm{~kg} \mathrm{~mol}^{-1}\right)$ in the surrounding bath containing $143 \mathrm{mM}$ sucrose. Based on the bulk measurements, the osmotic pressure of the polymer mixture containing $6 \mathrm{wt} \% \operatorname{PEG}\left(8 \mathrm{~kg} \mathrm{~mol}^{-1}\right)$ and $6.4 \mathrm{wt} \%$ dextran $\left(10 \mathrm{~kg} \mathrm{~mol}^{-1}\right)$ corresponds to roughly $100 \mathrm{mM}$ sucrose. Thus the immersion into $143 \mathrm{mM}$ sucrose-laden bath subjects the GUV population to a net hypertonic stress $(\Delta \Pi=R T \Delta c \approx 0.1 \mathrm{MPa})$, which acts to expel water from the vesicular interior and elevate polymer concentrations (see above).

31 Time-lapse fluorescence images reveal a sequence of four well-differentiated kinetic regimes described in 32 turn below. First, within tens of seconds after subjecting the GUV to the hyperosmotic stress, tubular 33 protrusions directed toward the interior of the vesicles become visible. This appearance of localized tubular 34 invaginations - in response to the reduction of enclosed volume for a fixed membrane area, which ensues 
1 because of osmotic removal of water - appears counter-intuitive. It is at variance with the theoretically

2 expected shape diagrams predicting increased membrane fluctuations and well-defined global shape

3 changes, which characterize the equilibrium configurations for various reduced volumes ( $\underline{56})$. It, however,

4 confirms similar findings reported previously $(\underline{42}, \underline{49}, \underline{57})$ and lends support to the notion that the tubular

5 protrusions are generated by in-situ changes and stabilized kinetically. Specifically, these studies show that

6 the initial membrane deformation into a nascent bud is prompted by an in-situ generated negative

7 spontaneous curvature because of the adsorption of PEG polymer to the inner leaflet of the membrane

8 surface (32). They further suggest that these nascent spherical buds grow via rapid flow of lipids producing

9 a string of buds, which relax into cylindrical tubes( $\underline{57})$.

11 Second, over the next several seconds $(100 \mathrm{~s}<\mathrm{t}<200 \mathrm{~s})$, the dextran-rich droplets suspended within the 12 vesicular lumen, display a gradual increase in the fluorescence intensity (due to the labeled dextran) and 13 grow in size (and polydispersity). The former is consistent with the droplet growth via diffusive transport 14 and the latter reflects coarsening. Monitoring the changes in the fluorescence intensity (due to labeled 15 dextran) of the droplets and the surrounding phase during this regime (Supplementary Information, Fig.

16 S2.1) reveals that the mean fluorescence intensity for all droplets continue to increase with time until 17 reaching an asymptotic intensity ( $\sim 4 \mathrm{~min})$. Quantifying the dextran partition coefficient $(K)$, defined as the 18 ratio of dextran in PEG-rich phase to dextran in dextran-rich phase, we estimate the asymptotic partition to 19 be $K \sim 0.26$, which is in good agreement with that observed for the bulk solution (Supplementary 20 Information, Fig. S2.1), and those reported previously (녀).

Analyzing the growth-coarsening rate of the droplets during this regime, we find a power-law dependence $(1, \underline{58})$. consistent with the generalized $R(t) \propto t^{\alpha}$ form with an exponent of $\alpha \cong 1.0( \pm 0.2), n=3$ (Fig. 2d). This is at variance with the universal scaling behaviors(59) that characterize growth and coarsening. The growth in three-dimensional bulk typically proceeds by diffusion-limited mechanisms, which correspond to $\alpha=1 / 2(\underline{60})$. Coarsening mechanisms, which depend on the volume fraction of the minority phase, are dominated by diffusion in the low volume-fraction limit, which produces discrete droplet. Here, the translational diffusive flux from smaller droplets to larger droplets facilitates the growth of the latter at the expense of the former via the so-called evaporation-condensation (Lifshitz-Slyozov-Wagner) mechanism in a manner akin to Ostwald ripening. This sets the growth exponent at $\alpha=1 / 3(\underline{61}, \underline{62})$. At intermediate volume fractions, coarsening occurs via collisions and coalescence of droplets undergoing the stochastic Brownian motion through the so-called Brownian-coagulation (Binder-Stauffer) mechanism ( $\underline{63}$,

$33 \underline{64})$. Both of these mechanisms set the growth exponent at $\alpha=1 / 3(\underline{61}, \underline{62})$. 
1 The observed linear growth represents a striking departure from the expected exponents for either the

2 diffusion-limited growth $(\alpha=1 / 2)$ or the droplet coarsening via either the Brownian coalescence or

3 Ostwald ripening mechanisms $(\alpha=1 / 3$ ) (see above). We suggest that this kinetic acceleration reflects the 4 roles proximal surface and the GUV confinement play in modulating transport processes. Indeed, previous

5 studies of surface-directed spinodal decomposition of polymer mixtures near surfaces and in capillaries $(\underline{5}$,

$6 \underline{\text { ) }}$ ) have similarly reported higher growth rates (as high as $\alpha=3 / 2$ ) and proposed theoretical explanations

7 based on the differences in the surface-wetting behaviors of the droplet- and the majority phases $(\underline{9}, \underline{65})$. In

8 the present case, the dextran-rich droplets almost invariably appear, grow, and coarsen deep within the

9 vesicular lumen away from the bounding membrane, with the latter remaining fully wetted by the PEG-

10 enriched majority phase during this regime. Based on the above, it appears reasonable that the depletion of

11 growing droplets from the bounding membrane act to increase the effective droplet concentration with time

12 leading to an apparent acceleration in coalescence kinetics.

14 Third, beyond roughly $200 \mathrm{~s}$, we find an abrupt interruption of the droplet coalescence process. Instead of 15 indefinite droplet growth culminating in macroscopic phase separation, we find that the extant droplets,

16 which were initially suspended within the vesicular interior, begin to accumulate at (and adhere to) the 17 membrane surface. Occassionally, we also find that some droplets become wrapped by the tubular 18 invaginations, which formed during the first regime (see above). In both cases, this partial "emulsification" 19 of the droplets by the membrane appears to be a key event for understanding the kinetic arrest of the droplet 20 coarsening. We reason that the adsorbed tubules at droplet surfaces can act as a barrier against the 21 coalescence of suspended droplets and limit the mobilities of peripheral droplets bound to the vesicular 22 boundary. Together, they arrest the coarsening process leading to a metastable multi-droplet organization 23 for the phase-separating polymers. Such dynamic arrests are common in many phase-separating systems 24 including colloids, gels, and concentrated protein solutions, but they typically arise when one of the phase 25 separating component gels or vitrifies producing non-equilibrium metastable states( $\underline{66})$. In the present case, 26 the dynamic arrest produces a rather unusual scenario: the morphological evolution emanating from an 27 unstable quench gives rise to metastable spatial organization of putative equilibrium phases (67) - the latter 28 confirmed by the observations of comparable intensities (within 4\%) of bound droplets.

30 From the vantage point of the membrane, the trafficking of dextran-rich droplets to the membrane surface 31 gives rise to a novel, complete-to-partial, wetting transition $(\underline{41}, \underline{48})$ in which the membrane surface, which 32 was initially wetted by the majority PEG-rich phase exclusively, becomes wetted by both co-existing 33 phases. At a basic level, this wetting transition reflects a changing balance between three relevant interfacial 
1 interactions(41): $\sigma_{p m}, \sigma_{d m}$, and $\sigma_{p d}$, which represent the interfacial tensions associated with the interfaces

2 between the PEG-rich phase $(p)$, the dextran-rich phase $(d)$, and the membrane $(m)$. During the early stages

$3(t<200 s)$ - when the dextran-rich droplets are growing by accretion of dextran molecules and have yet

4 to achieve equilibrium composition (see above) - the interfacial tension $\sigma_{p d}$ is still low, and the membrane

5 remains wetted by the PEG-enriching phase $\left(\sigma_{p d}<\sigma_{d m}-\sigma_{p m}\right)$. Over time, as growth due to molecular

6 exchanges enrich the droplets and deplete the bath in terms of dextran concentrations, $\sigma_{p d}$ continues to rise

7 and crosses $(t \cong 200 s)$ a threshold $\left(\sigma_{p d} \cong \sigma_{d m}-\sigma_{p m}\right)$, beyond which the energetic cost of wetting

8 the membrane by the dextran-rich droplet is smaller than the gain obtained by the lowering of the interfacial

9 area between the two aqueous phases.

11 Fourth, upon wetting the membrane surface, the dextran-rich droplets deform the wetted area producing a 12 striking mosaic of outwardly directed buds, which remain in contiguity with the mother vesicle (Fig. 2b13 c). These buds thus provide specialized niches to spatially organize intravesicular aqueous phases and 14 divide the otherwise contiguous membrane of nominally single composition into distinct segments with 15 different curvatures and mechanical tensions. The sizes (and the numbers) of the buds correspond to those 16 of individual droplets formed at the onset of membrane wetting $t \cong 250 \mathrm{~s}$. Although similar in 17 appearance $(\underline{68}, \underline{69})$, this droplet-induced budding is distinctly different from the budding transitions in 18 phase-separated membranes, $(\underline{68}, \underline{70})$. Unlike domain-mediated budding, which is induced by the 19 thermodynamic drive to minimize line tension in molecularly differentiated domains, the buds produced by 20 the coarsening arrest in the present case are compositionally indistinguishable from the surrounding 21 membrane and present little or no line tension.

23 From a mechanics-energetics point of view, the domain-induced budding is determined by the balance of 24 line tension, $(\sigma)$, that exists at the domain boundary and the curvature energy $(\kappa)-$ both determined by 25 the membrane's internal compositional degrees of freedom: Above a characteristic length, $l \cong \kappa / \sigma$, 26 deformation of an initially flat domain producing buds is energetically favored. (70) Using typical values 27 of $\kappa \sim 10^{-19} \mathrm{~J}$ and $\sigma \sim 10^{-11}-10^{-12} \mathrm{~N}$ then estimates the critical threshold of 10-100 $\mathrm{nm}$ for the hemispherical 28 bud to form. In contrast, the energetic driver for the droplet-induced budding has an extrinsic origin: the interfacial tension between the co-existing aqueous phases, namely dextran-rich droplets and the PEG-rich surrounding, which does not depend on the membrane properties. Here, the competition between the

31 corresponding interfacial energy $\sigma_{p d} \times A_{p d}$ and the curvature energy sets the characteristic length scale at $\sqrt{\kappa / \sigma_{p d}}$. Using the typical values of $\kappa \sim 10^{-19} \mathrm{~J}$ and our previous estimate of $\sigma_{p d} \sim 0.36 \times 10^{-3} \mathrm{~N} . \mathrm{m}^{-1}(\underline{71})$

33 for comparable compositions suggests that, here too, budding is favored beyond the nanometer scale droplet 
size of $\sim 10 \mathrm{~nm}$. Moreover, because the aqueous phase interfacial tension can be tuned by changing polymer

2 types, concentrations, and molecular weights (뇨), droplet-induced budding morphologies are determined

3 independent of membrane composition.

5 Next, it is notable that the bud shapes, sizes, and numbers do not change for several hours ( $\sim 4$ hrs).

6 Examining the slow evolution of the bud morphology over the long-term $(>24 \mathrm{~h})$ reveals a slow coarsening,

7 which ultimately replaces the multi-budded GUV configuration with one characterized by a single bud

8 (Supplementary Information, Fig. S2.2), such as expected at equilibrium(49). This dramatic slow-down

9 in the coarsening of membrane-bound dextran-rich droplets parallels the similarly slow coarsening

10 dynamics of domain-induced buds $(69,72-74)$ : As two membrane-bound droplets, which locally deform

11 the membrane approach one another, the membrane segment ("skirt" region) between the droplets is also

12 deformed (Supplementary Information, Fig. S2.2). When the droplets are within the ranges of their

13 elastic decay lengths, this deformation of the surrounding region gives rise to a net inter-bud repulsive

14 force and elevates the activation energy barrier for bud coalescence $(69,73,75)$. The reasoning above then

15 suggests that the the elastic properties of the membrane arrest the droplet coarsening - a direct consequence

16 of preferential interactions between the membrane and phase-separated droplets. Another consequence of

17 this short length scale inter-bud repulsion can spatially organize membrane domains producing higher-order

18 superlattice patterns. Although the polydispersity of buds and our measurement methods are not readily

19 amenable to detailed quantitative characterization, we often observe relatively monodisperse population of

20 buds that organize into extended quasi-ordered two-dimensional lattices (Supplementary Information

$21 \quad$ Fig. S2.3)

23 Both the liquid-liquid phase separation and budding above are fully reversible, albeit the reversal kinetics

24 are different. Upon lowering the sucrose concentration in the bath close to the starting isotonic value, the

25 phase-separated interior of the budded GUVs abruptly transitions to the the single homogeneous phase and

26 the membrane boundary unbuds, restoring the initial spherical shape (Supplementary Video S2 and

27 Supplementary Fig. S2.4). This ability of the membrane buds to robustly sequester their content (i.e., 28 dextran) as a distinct phase and subsequently redeliver such as by an osmotically induced phase transition 29 appears curious. The former suggest a potential physical mechanism by which buds might select their 30 molecular cargoes, such as in the formation of transport vesicles $(\underline{76})$. The latter appears 31 phenomenologically similar to fusion of vesicles carrying different cargos(77).

33 Systematic mapping of the phase separation trajectory for a range of different weight fractions of PEG and 34 dextran at a fixed total polymer concentration reveals that the essential dynamics above remain largely 
unchanged (Fig. 3a, Supplementary Fig. S2.5 and Supplementary Video S3-S6). At low dextran

2 concentrations, droplet sizes (and correspondingly the buds) are proportionately smaller, and conversely

3 elevated dextran concentrations yields larger droplets and buds. In the limiting cases, the smallest

4 membrane-bound buds produced at the lowest dextran concentrations merely decorate the membrane

5 without budding (Supplementary Video S3 and S4) and at the highest dextran concentrations tested, the

6 reversal of the majority and droplet phases produce single buds encapsulating the minority PEG phase with

7 the majority dextran phase wetting the mother vesicle (Supplementary Video S6).

9 The phase separation in the PEG-Dextran ATPS system is known to depend on the molecular weights of 10 the two phase-separating components. Higher molecular weight polymers (1) depress the binodal to lower 11 concentrations, thus favouring phase separation; (2) lengthen the tie-lines, segregating the polymers more 12 effectively; and (3) increase the magnitude of interfacial tension( $\underline{30})$. These effects become more 13 pronounced when the ratio of the molecular weight of the two polymers is also large( $\underline{78})$. To characterize 14 how the better segregation of polymers and higher interfacial tension influence the kinetic interplay of 15 droplet coarsening and budding, we repeated the experiments by substituting dextran $\left(10 \mathrm{~kg} \cdot \mathrm{mol}^{-1}\right)$ by 16 dextran $450\left(\mathrm{~kg} \mathrm{~mol}^{-1}\right)$. We find that the droplets coarsen to larger diameters, often producing single large 17 droplets, before adhering to the membrane (Fig. 3b, Supplementary Video S7). This is not surprising since 18 elevated interfacial tension between the incipient co-existing phases increase the thermodynamic driving 19 force for droplet coalescence allowing rapid equilibration of the ATPS phases within the vesicular lumen prior to budding. Subsequently, single large droplets bud, producing morphologies similar to those typically obtained by slow and step-wise approach to phase separation under near-equilibrium conditions(49).

In all of the experiments above, membrane composition contained a finite proportion of PEG-derivatized lipid (DOPE-mPEG , 2.2 mol\%), which is incorporated to facilitate GUV formation. These PEG-displaying dopants, however, also act to preferentially wet the membrane by the incipient PEG-rich phase (and allowing the dextran-rich phase to nucleate, grow, and coarsen within the vesicular interior) during phase separation. To explore the effect of reversing the membrane preference for the wetting phase, we replaced DOPE-mPEG by a glycolipid GM1, which interacts preferentially with the dextran-rich phase (see methods). Remarkably, we find a complete and a drastic reversal in the growth pattern (Fig. 3c,

30 Supplementary Video S8): The dextran-droplets almost exclusively nucleate, grow, and coarsen at the 31 membrane surface - suggesting a shift from homogeneous to heterogeneous nucleation - likely by spatially 32 localizing compositional fluctuations needed for phase separation in the vicinity of the membrane boundary. 
1 Cumulated weight of the results presented above reveals the complex and dynamic interplay between the

2 membrane boundary and the liquid-liquid phase separation inside giant vesicles altering equilibration

3 pathways and stabilizing kinetically formed morphologies.

5 This dynamic relationship between phase separation in the aqueous bulk and the interfacial wetting-induced

6 budding at the membrane surface raises an interesting question: How do the membrane's compositional

7 degrees of freedom influence this interplay? In the cellular context, this simple question has important

8 parallels: How do living cells control sizes, shapes, distributions, and other physical properties of the droplet

9 condensates? What roles do membrane play in regulating LLPS inside the cell? How is the LLPS activity

10 communicated to the membrane interface, where many signaling events occur? Recent studies highlight

11 several biophysical pathways, such as the roles membranes play in (1) reducing concentration thresholds

12 for LLPS by elevating local protein concentrations $(\underline{17}, \underline{20}, \underline{79})$ and (2) facilitating nucleation and growth of

13 membrane-bound protein condensates such as seen most prominently in T-cell signaling where activation

14 of T-cell receptor at the membrane surface triggers multi-phosphorylation of LAT, which in turn recruits

15 additional proteins, Grb2 and divalent SOS, driving RAS activating(21) condensate formation at the 16 membrane surface $(\underline{80})$. Yet little is known about the relations between two-dimensional lipid-lipid phase

17 separation and membrane interactions of condensates produced by the three-dimensional LLPS.

To investigate whether the membrane association of dextran-rich liquid droplets can dynamically activate membrane's compositional degrees of freedom, we replaced single membrane lipids of our GUVs with a ternary mixture containing an equimolar concentration of POPC, cholesterol, and sphingomyelin (2:2:1). To induce droplet formation in real-time, we encapsulated an aqueous mixture containing $6 \mathrm{wt} \%$ PEG (8 $\left.\mathrm{kg} \mathrm{mol}^{-1}\right)$ and $6.4 \mathrm{wt} \%$ dextran $\left(10 \mathrm{~kg} \mathrm{~mol}^{-1}\right)$ inside the GUVs and subjected them to a hypertonic stress from the bath containing $143 \mathrm{mM}$ sucrose. Time-lapse fluorescence microscopy images confirm the general phase ordering trajectory described above (Fig. 4, Supplementary Video S9). Additionally, they reveal a remarkable redistribution of membrane components: Upon droplet-induced membrane budding, the initial uniform distribution of the two phase-sensitive dyes in the membranes of the GUVs, reflecting a single homogeneous phase, is abandoned, replaced by a pattern of membrane fluorescence - fully commensurate with the pattern of droplet-induced buds: Each bud is capped by the liquid-ordered $\left(\mathrm{L}_{0}\right)$ lipid phase enriched in cholesterol and sphingomyelin and the skirt region between the buds is occupied by the phospholipid31 rich liquid-disordered $\left(\mathrm{L}_{\mathrm{d}}\right)$ phase.

33 There are several salient features of this emergent lipid phase pattern, which matches the underling droplet 34 pattern, all of which can be readily understood in terms of well-known principles of membrane mechanics, 
1 dynamics, and thermodynamics. First, perhaps the most remarkable aspect of our observations is the 2 appearance of co-existing phases in direct correspondence with the emergence of droplet-induced 3 membrane budding. Similar isothermal phase transitions have been previously observed, in the absence of

4 specific lipid-lipid or lipid-protein interactions, when multi-component lipid bilayers are subject to 5 gradients of (1) curvatures, such as occurs when tubes are pulled from giant vesicles( $\underline{81} \underline{82})$; (2) mechanical tension( $\underline{83})$, such as produced by osmotic stresses $(\underline{83}, \underline{84})$; or (3) fluctuations, such as modulated by adhesion $(\underline{85}, \underline{86})$. In all of these cases, the plunge into phase co-existence occurs most reliably when membrane compositions are in the vicinity of miscibility phase transition $(\underline{82,} \underline{87})$. In the present case, droplet wetting of the membrane locally renders the membrane tense, suppresses thermal undulations, and produces curvature gradients in the neck region separating the bud from the mother vesicle. Together, these mechanical-chemical perturbations provide a basis for understanding the observed lateral phase separation

Second, it is notable that the phase-separated domains are spatially commensurate with the underlying spatial patterns of membrane morphology and intravesicular polymer-enriched droplets. The lateral sorting of domains - albeit existing ones - in spatially-defined curvature niches, within single membranes have been observed in many independent contexts and over many different length scales( $\underline{88})$. A separate mechanism for similar domain alignment involves the preferential spatial localization of specific molecules, which are differentially solubilized between the two co-existing phases $(\underline{86}, \underline{89})$. Indeed, a previous study by one of us(9) found that preferential interaction of a molecule, i.e., a PEG-terminated saturated lipid, solubilized preferentially in the Lo phase with the PEG-rich phase in the vesicular interior was sufficient to induce spatial ordering of pre-existing membrane domains in registry with the intravesicular bulk. In this same vein, the small concentration of Ld-phase partitioning DOPE-mPEG in the present study can readily explain the spatial positioning of the incipient membrane phases. Together, these considerations may explain our observations of simultaneous lipid phase separation and spatial localization of membrane domains driving an exquisite coupling of three-dimensional liquid-liquid phase separation within the vesicular interior with the lateral phase separation of lipids at the membrane boundary.

Collectively, the results presented here exemplify the temporal morphological evolution during the segregative phase ordering of a mixture of polymers inside giant vesicles following an abrupt quench. Our results could shed light on non-equilibrium behaviours of liquid-liquid phase separation in cell-like compartments. The crowded nature of the cytoplasm; physical confinement in small volumes; and

33 interactions with the proximal membrane, which is at once flexible, deformable, and heterogeneous can all 34 be expected to influence the phase transition trajectories of induced liquid-liquid phase separation. It can 
1 dynamically arrest phase separation, produce metastable spatial organization of co-existing phases, and 2 couple the liquid-liquid phase separation in the 3D interior with the $2 \mathrm{D}$ lipid-lipid phase separation at the 3 membrane boundary - potentially suggesting a general physical-chemical principle that can drive 4 mesoscale functional organization of membraneless organelles in living and artificial cells. 
References Cited.

1. A. J. Bray, Theory of phase-ordering kinetics. Advances in Physics 43, 357-459 (1994).

2. K. Binder, Collective diffusion, nucleation, and spinodal decomposition in polymer mixtures. J. Chem. Phys. 79, 6387-6409 (1983).

3. A. Onuki, Phase Transition Dynamics. (Cambridge University Press, Cambridge, 2002).

4. K. Binder, Spinodal decomposition in confined geometry. J. Non-Equilib. Thermodyn. 23, 1-44 (1998).

5. H. Tanaka, Interplay between wetting and phase separation in binary fluid mixtures: roles of hydrodynamics. Journal of Physics-Condensed Matter 13, 4637-4674 (2001).

6. S. J. Puri, Surface-directed spinodal decomposition. Journal of Physics-Condensed Matter 17, R101R142 (2005).

7. R. A. L. Jones, L. J. Norton, E. J. Kramer, F. S. Bates, P. Wiltzius, Surface-directed spinodal decomposition. Physical Review Letters 66, 1326-1329 (1991).

8. P. Wiltzius, A. Cumming, Domain growth and wetting in polymer mixtures. Physical Review Letters 66, 3000-3003 (1991).

9. S. M. Troian, Coalescence induced domain growth near a wall during spinodal decomposition. Physical Review Letters 71, 1399-1402 (1993).

10. H. Tanaka, Double-phase separation in a confined, symmetrical binary mixture - interface quench effect unique to bicontinuous phase-separation. Physical Review Letters 72, 3690-3693 (1994).

11. J. Berry, C. P. Brangwynne, M. Haataja, Physical principles of intracellular organization via active and passive phase transitions. Rep. Prog. Phys. 80, 42 (2018).

12. A. A. Hyman, C. A. Weber, F. Juelicher, in Annual Review of Cell and Developmental Biology, Vol 30, R. Schekman, R. Lehmann, Eds. (Annual Reviews, Palo Alto, 2014), vol. 30, pp. 39-58.

13. S. Alberti, Phase separation in biology. Current Biology 27, R1097-R1102 (2017).

14. S. Alberti, The wisdom of crowds: regulating cell function through condensed states of living matter. Journal of Cell Science 130, 2789-2796 (2017).

15. S. Alberti, A. Gladfelter, T. Mittag, Considerations and Challenges in Studying Liquid-Liquid Phase Separation and Biomolecular Condensates. Cell 176, 419-434 (2019).

16. S. F. Banani, H. O. Lee, A. A. Hyman, M. K. Rosen, Biomolecular condensates: organizers of cellular biochemistry. Nature Reviews Molecular Cell Biology 18, 285-298 (2017).

17. P. L. Li et al., Phase transitions in the assembly of multivalent signalling proteins. Nature 483, 336U129 (2012).

18. Y. Shin, C. P. Brangwynne, Liquid phase condensation in cell physiology and disease. Science 357 , (2017).

19. M. L. Zeng et al., Reconstituted Postsynaptic Density as a Molecular Platform for Understanding Synapse Formation and Plasticity. Cell 174, 1172 (2018).

20. S. Banjade, M. K. Rosen, Phase Transitions of Multivalent Proteins Can Promote Clustering of Membrane Receptors. eLife 3, 68 (2014).

21. W. Y. C. Huang et al., A molecular assembly phase transition and kinetic proofreading modulate Ras activation by SOS. Science 363, 1098 (2019).

22. S. F. Fenz, K. Sengupta, Giant vesicles as cell models. Integrative Biology 4, 982-995 (2012).

23. M. Li, X. Huang, T. Y. D. Tang, S. Mann, Synthetic cellularity based on non-lipid micro-compartments and protocell models. Current Opinion in Chemical Biology 22, 1-11 (2014).

24. M. Weiss et al., Sequential bottom-up assembly of mechanically stabilized synthetic cells by microfluidics. Nature Materials 17, 89 (2018).

25. W. J. Mu et al., Membrane-confined liquid-liquid phase separation toward artificial organelles. Sci. Adv. 7, 9 (2021).

26. P.-Å. Albertsson, in Advances in Protein Chemistry, J. T. E. C.B. Anfinsen, M. R. Frederic, Eds. (Academic Press, 1970), vol. Volume 24, pp. 309-341.

27. S. S. Ribeiro, N. Samanta, S. Ebbinghaus, J. C. Marco, The synergic effect of water and biomolecules in intracellular phase separation. Nature Reviews Chemistry 3, 552-561 (2019). 
28. A. Gustafsson, H. Wennerstrom, F. Tjerneld, The nature of phase-separation in aqueous 2-polymer systems. Polymer 27, 1768-1770 (1986).

29. B. Y. Zaslavsky, V. N. Uversky, In Aqua Veritas: The Indispensable yet Mostly Ignored Role of Water in Phase Separation and Membrane-less Organelles. Biochemistry 57, 2437-2451 (2018).

30. D. Forciniti, C. K. Hall, M. R. Kula, Interfacial-tension of polyethyleneglycol-dextran-water systems influence of temperature and polymer molecular-weight. Journal of Biotechnology 16, 279-296 (1990).

31. Y. G. Liu, R. Lipowsky, R. Dimova, Concentration Dependence of the Interfacial Tension for Aqueous Two-Phase Polymer Solutions of Dextran and Polyethylene Glycol. Langmuir 28, 3831-3839 (2012).

32. Y. G. Liu, R. Lipowsky, R. Dimova, Giant Vesicles Encapsulating Aqueous Two-Phase Systems: From Phase Diagrams to Membrane Shape Transformations. Frontiers in Chemistry 7, (2019).

33. H. X. Zhou, G. N. Rivas, A. P. Minton, in Annual Review of Biophysics. (Annual Reviews, Palo Alto, 2008), vol. 37, pp. 375-397.

34. F. M. Menger, M. I. Angelova, Giant vesicles: Imitating the cytological processes of cell membranes. Accounts of Chemical Research 31, 789-797 (1998).

35. P. Walde, K. Cosentino, H. Engel, P. Stano, Giant Vesicles: Preparations and Applications. Chembiochem 11, 848-865 (2010).

36. C. Faure et al., Modeling leakage kinetics from multilamellar vesicles for membrane permeability determination: Application to glucose. Biophysical Journal 91, 4340-4349 (2006).

37. D. W. Deamer, J. Bramhall, Permeability of lipid bilayers to water and ionic solutes. Chem. Phys. Lipids 40, 167-188 (1986).

38. M. Mally, J. Majhenc, S. Svetina, B. Zeks, Mechanisms of equinatoxin II-induced transport through the membrane of a giant phospholipid vesicle. Biophysical Journal 83, 944-953 (2002).

39. M. R. Helfrich, L. K. Mangeney-Slavin, M. S. Long, Y. Djoko, C. D. Keating, Aqueous phase separation in giant vesicles. Journal of the American Chemical Society 124, 13374-13375 (2002).

40. C. D. Keating, Aqueous Phase Separation as a Possible Route to Compartmentalization of Biological Molecules. Accounts of Chemical Research 45, 2114-2124 (2012).

41. Y. H. Li, R. Lipowsky, R. Dimova, Transition from complete to partial wetting within membrane compartments. Journal of the American Chemical Society 130, 12252-+ (2008).

42. Y. H. Li, R. Lipowsky, R. Dimova, Membrane nanotubes induced by aqueous phase separation and stabilized by spontaneous curvature. Proc. Natl. Acad. Sci. U. S. A. 108, 4731-4736 (2011).

43. M. S. Long, C. D. Jones, M. R. Helfrich, L. K. Mangeney-Slavin, C. D. Keating, Dynamic microcompartmentation in synthetic cells. Proc. Natl. Acad. Sci. U. S. A. 102, 5920-5925 (2005).

44. M. S. Long, A.-S. Cans, C. D. Keating, Budding and asymmetric protein microcompartmentation in giant vesicles containing two aqueous phases. Journal of the American Chemical Society 130, 756-762 (2008).

45. M. Andes-Koback, C. D. Keating, Complete Budding and Asymmetric Division of Primitive Model Cells To Produce Daughter Vesicles with Different Interior and Membrane Compositions. Journal of the American Chemical Society 133, 9545-9555 (2011).

46. R. Dimova, R. Lipowsky, Lipid membranes in contact with aqueous phases of polymer solutions. Soft Matter 8, 6409-6415 (2012).

47. Y. Li, R. Lipowsky, R. Dimova, Membrane nanotubes induced by aqueous phase separation and stabilized by spontaneous curvature. Proc. Natl. Acad. Sci. U. S. A. 108, 4731-4736 (2011).

48. H. Kusumaatmaja, Y. Li, R. Dimova, R. Lipowsky, Intrinsic Contact Angle of Aqueous Phases at Membranes and Vesicles. Physical Review Letters 103, (2009).

49. R. Dimova, R. Lipowsky, Giant Vesicles Exposed to Aqueous Two-Phase Systems: Membrane Wetting, Budding Processes, and Spontaneous Tubulation. Advanced Materials Interfaces 4, (2017).

50. N. F. Morales-Penningston et al., GUV preparation and imaging: Minimizing artifacts. Biochimica Et Biophysica Acta-Biomembranes 1798, 1324-1332 (2010).

51. S. L. Veatch, S. L. Keller, Miscibility phase diagrams of giant vesicles containing sphingomyelin. Physical Review Letters 94, 4 (2005). 
52. S. Sriwongsitanont, M. Ueno, Effect of a PEG lipid (DSPE-PEG2000) and freeze-thawing process on phospholipid vesicle size and lamellarity. Colloid Polym. Sci. 282, 753-760 (2004).

53. L. M. Dominak, C. D. Keating, Polymer encapsulation within giant lipid vesicles. Langmuir 23, $7148-$ 7154 (2007).

54. T. Baumgart, G. Hunt, E. R. Farkas, W. W. Webb, G. W. Feigenson, Fluorescence probe partitioning between L-o/L-d phases in lipid membranes. Biochimica Et Biophysica Acta-Biomembranes 1768, 2182-2194 (2007).

55. R. Hatti-Kaul. (Humana Press, Totowa, N.J., 2000).

56. U. Seifert, Configurations of fluid membranes and vesicles. Advances in Physics 46, 13-137 (1997).

57. Y. G. Liu, J. Agudo-Canalejo, A. Grafmuller, R. Dimova, R. Lipowsky, Patterns of Flexible Nanotubes Formed by Liquid-Ordered and Liquid-Disordered Membranes. ACS Nano 10, 463-474 (2016).

58. H. Furukawa, A dynamic scaling assumption for phase-separation. Advances in Physics 34, 703-750 (1985).

59. P. C. Hohenberg, B. I. Halperin, Theory of dynamic critical phenomena. Reviews of Modern Physics 49, 435-479 (1977).

60. A. J. Bray, Coarsening dynamics of phase-separating systems. Philos. Trans. R. Soc. A-Math. Phys. Eng. Sci. 361, 781-791 (2003).

61. I. M. Lifshitz, V. V. Slyozov, The kinetics of precipitation from supersaturated solid solutions. Journal of Physics and Chemistry of Solids 19, 35-50 (1961).

62. P. W. Voorhees, Ostwald ripening of 2-phase mixtures. Annual Review of Materials Science 22, $197-$ 215 (1992).

63. K. Binder, D. Stauffer, Theory for slowing down of relaxation and spinodal decomposition of binarymixtures. Physical Review Letters 33, 1006-1009 (1974).

64. K. Binder, D. Stauffer, Statistical-theory of nucleation, condensation and coagulation. Advances in Physics 25, 343-396 (1976).

65. P. Keblinski, W. J. Ma, A. Maritan, J. Koplik, J. R. Banavar, Domain growth near a wall in spinodal decomposition. Physical Review Letters 72, 3738-3738 (1994).

66. P. J. Lu et al., Gelation of particles with short-range attraction. Nature 453, 499-U494 (2008).

67. A. Stradner et al., Equilibrium cluster formation in concentrated protein solutions and colloids. Nature 432, 492-495 (2004).

68. T. Baumgart, S. T. Hess, W. W. Webb, Imaging coexisting fluid domains in biomembrane models coupling curvature and line tension. Nature 425, 821-824 (2003).

69. M. Yanagisawa, M. Imai, T. Masui, S. Komura, T. Ohta, Growth dynamics of domains in ternary fluid vesicles. Biophysical Journal 92, 115-125 (2007).

70. F. Julicher, R. Lipowsky, Domain-induced budding of vesicles. Physical Review Letters 70, 2964-2967 (1993).

71. F. P. Cakmak, C. D. Keating, Combining Catalytic Microparticles with Droplets Formed by Phase Coexistence: Adsorption and Activity of Natural Clays at the Aqueous/Aqueous Interface. Scientific Reports 7, 14 (2017).

72. S. Semrau, T. Idema, T. Schmidt, C. Storm, Membrane-Mediated Interactions Measured Using Membrane Domains. Biophysical Journal 96, 4906-4915 (2009).

73. T. S. Ursell, W. S. Klug, R. Phillips, Morphology and interaction between lipid domains. Proc. Natl. Acad. Sci. U. S. A. 106, 13301-13306 (2009).

74. M. Laradji, P. B. Sunil Kumar, Dynamics of domain growth in self-assembled fluid vesicles. Physical Review Letters 93, 4 (2004).

75. M. Laradji, P. B. Kumar, Anomalously slow domain growth in fluid membranes with asymmetric transbilayer lipid distribution. Physical Review E 73, (2006).

76. M. J. Kuehn, J. M. Herrmann, R. Schekman, COPII-cargo interactions direct protein sorting into ERderived transport vesicles. Nature 391, 187-190 (1998).

77. J. S. Bonifacino, B. S. Glick, The mechanisms of vesicle budding and fusion. Cell 116, 153-166 (2004). 
78. D. Forciniti, C. K. Hall, M. R. Kula, Influence of polymer molecular-weight and temperature on phasecomposition in aqueous 2-phase systems. Fluid Phase Equilib. 61, 243-262 (1991).

79. L. B. Case, J. A. Ditlev, M. K. Rosen, in Annual Review of Biophysics, Vol 48, K. A. Dill, Ed. (2019), vol. 48, pp. 465-494.

80. X. L. Su et al., Phase separation of signaling molecules promotes T cell receptor signal transduction. Science 352, 595-599 (2016).

81. U. Seifert, Curvature-induced lateral phase segregation in 2-component vesicles. Physical Review Letters 70, 1335-1338 (1993).

82. A. Roux et al., Role of curvature and phase transition in lipid sorting and fission of membrane tubules. Embo J. 24, 1537-1545 (2005).

83. K. Oglecka, P. Rangamani, B. Liedberg, R. S. Kraut, A. N. Parikh, Oscillatory phase separation in giant lipid vesicles induced by transmembrane osmotic differentials. eLife 3, (2014).

84. T. Hamada, Y. Kishimoto, T. Nagasaki, M. Takagi, Lateral phase separation in tense membranes. Soft Matter 7, 9061-9068 (2011).

85. V. D. Gordon, M. Deserno, C. M. J. Andrew, S. U. Egelhaaf, W. C. K. Poon, Adhesion promotes phase separation in mixed-lipid membranes. Epl 84, 6 (2008).

86. J. Zhao, J. Wu, S. L. Veatch, Adhesion Stabilizes Robust Lipid Heterogeneity in Supercritical Membranes at Physiological Temperature. Biophysical Journal 104, 825-834 (2013).

87. A. Tian, T. Baumgart, Sorting of Lipids and Proteins in Membrane Curvature Gradients. Biophysical Journal 96, 2676-2688 (2009).

88. A. Callan-Jones, B. Sorre, P. Bassereau, Curvature-Driven Lipid Sorting in Biomembranes. Cold Spring Harbor Perspectives in Biology 3, (2011).

89. A. S. Cans, M. Andes-Koback, C. D. Keating, Positioning lipid membrane domains in giant vesicles by micro-organization of aqueous cytoplasm mimic. Journal of the American Chemical Society 130, 74007406 (2008). 
Figures

Liquid-Liquid Phase Separation inside Giant Vesicles Drives Shape Deformations and Induces

\section{Lipid Membrane Phase Separation}

Wan-Chih $\mathrm{Su}^{1}$, Doug L. Gettel ${ }^{2}$, Andrew T. Rowland ${ }^{3}$, Christine D. Keating*,3 Atul N. Parikh*1,2,4

${ }^{1}$ Chemistry, ${ }^{2}$ Chemical Engineering, and ${ }^{4}$ Biomedical Engineering Graduate Programs, University of California, Davis, California 95616, USA

${ }^{3}$ Department of Chemistry, The Pennsylvania State University, University Park, PA 16802, USA 
a
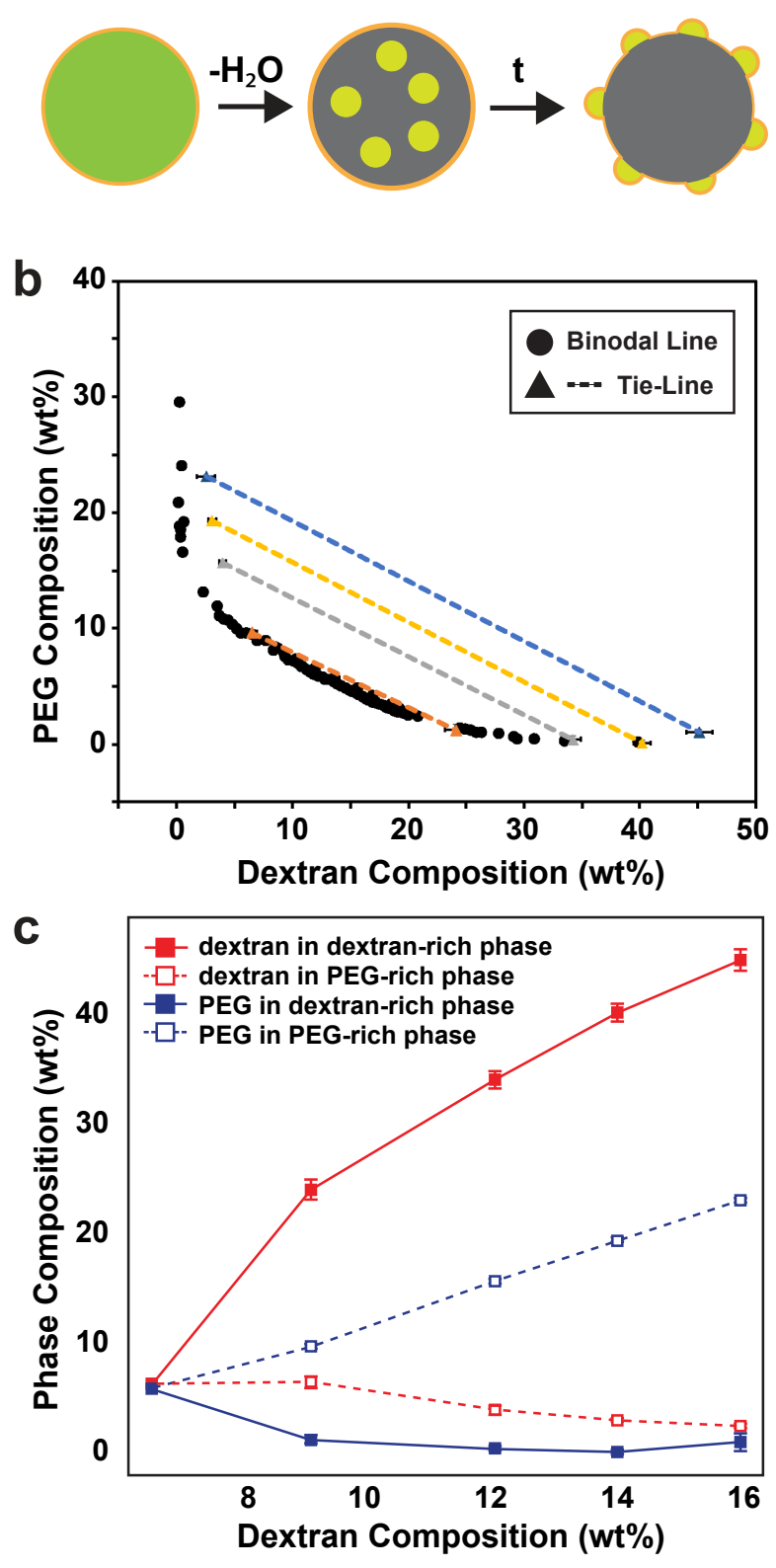

Figure 1. (a) Schematics of the osmotic deswelling pathways for inducing liquid-liquid phase separation in situ.; (b) Binodal and tie lines of PEG $8 \mathrm{~kg} \mathrm{~mol}^{-1}$ and dextran $10 \mathrm{~kg} \mathrm{~mol}^{-1}$ at room temperature. Binodal obtained by cloud-point method in bulk solution. Tie-lines obtained from the phase compositions of PEG-rich and dextran-rich phase. The dextran concentration of initial solutions (in a ratio of PEG to dextran equal to 16 to 15 ) are (1) $16 \mathrm{wt} \%(\triangle)$; (2) $14 \mathrm{wt} \%$ $(\triangle)$; (3) $12 \mathrm{wt} \%(\triangle)$; (4) $9 \mathrm{wt} \%(\triangle)$. The end points of tie-lines represent the composition of the PEG-rich phase (upper left point) and dextran-rich phase (lower right point). (c) Measurement of phase composition of polymers in PEG-rich phase and dextran-rich phase. 


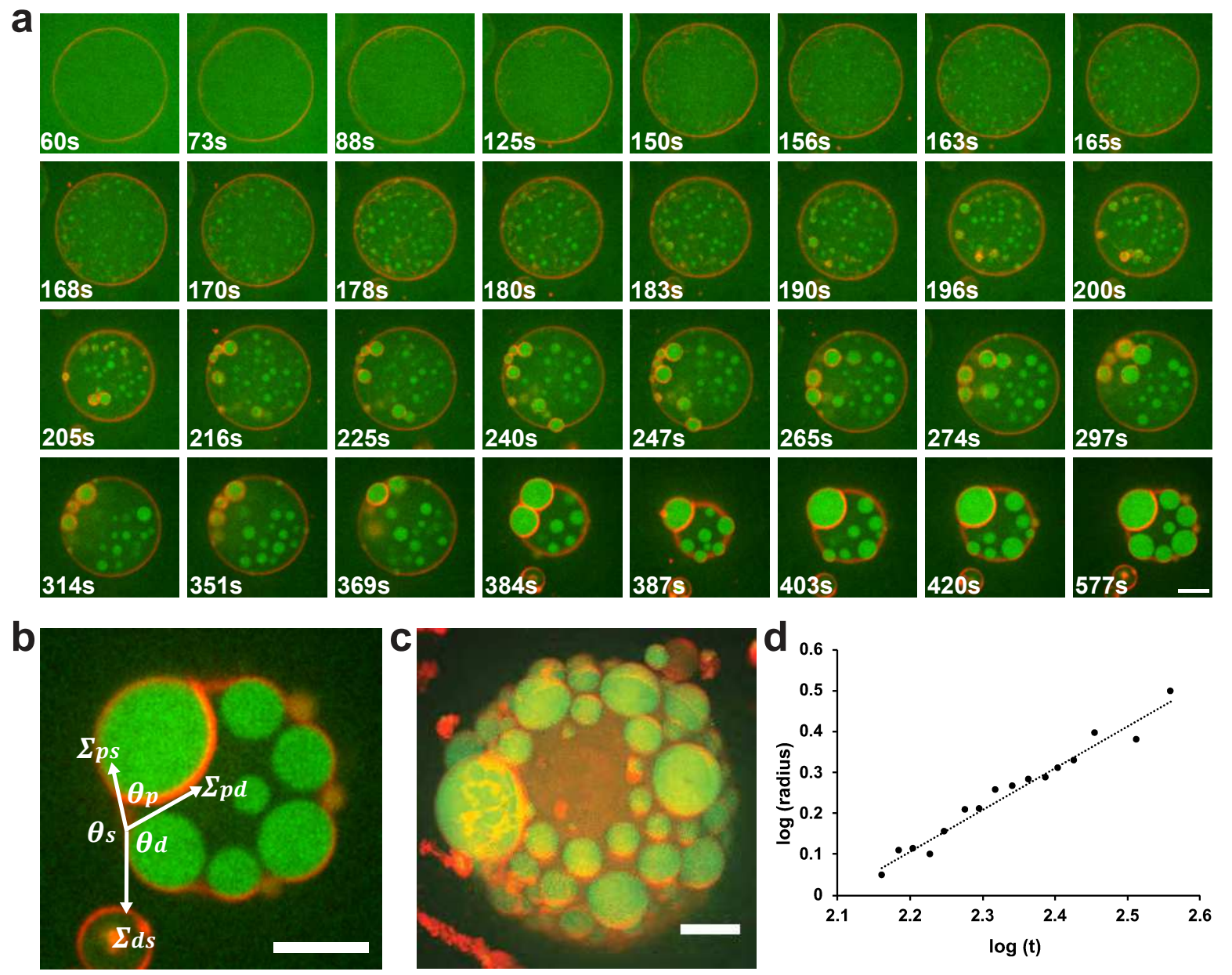

Figure 2. (a) Selected frames and (c) 3D image from a time-lapse movie (Supplementary Video S1) of confocol fluorescence microscopy images of GUVs encapsulating a mixture of $6.0 \%$ (w/w) PEG $8 \mathrm{~kg} \mathrm{~mol}^{-1}$ and 6.4\% (w/w) dextran $10 \mathrm{~kg} \mathrm{~mol}^{-1}$ doped with $0.001 \%$ AlexaFluor ${ }^{\circledR}$ 488-Dextran upon immersion in $143 \mathrm{mM}$ sucrose solution. (b) Droplet shape characterization by three contact angle $\theta_{p}\left(74.08^{\circ}\right), \theta_{d}\left(120.58^{\circ}\right)$, and $\theta_{s}\left(165.28^{\circ}\right)$ corresponding to PEGrich phase, dextran-rich phase and exterior solution. $\Sigma_{p s}, \Sigma_{p d}$, and $\Sigma_{d s}$ represents three tensions, which can be calculated based on known value and contact angles, along three interfaces (i.e. PEG-rich phase and dextran-rich phase, $\Sigma_{p d}$; PEG-rich phase and external solution, $\Sigma_{p s}$; and dextran-rich phase and external solution, $\left.\Sigma_{d s}\right) \underline{1}$; (d) Dextran droplet growth rate $(\mathrm{n}=3)$. Droplet growth follows a universal scaling behavior $\underline{2}$ in which the dominant length scale $(R(t))$ of the new phase grows with time: $\log (R(t)) \propto \log (t)$. The plot showing $\log (\mathrm{r})$ vs $\log (\mathrm{t})$ analysis of dextran droplets give rise to a linear equation of droplet growth: $\log (r)=$ $1.0249 \times \log (t)-2.1487, R^{2}=0.9671$. All GUVs imaged consist of a mixture containing 96.8\% POPC, 2.2\% DOPE-mPEG, 1\% Rho-DOPE. Scale bar, $10 \mu \mathrm{m}$. 


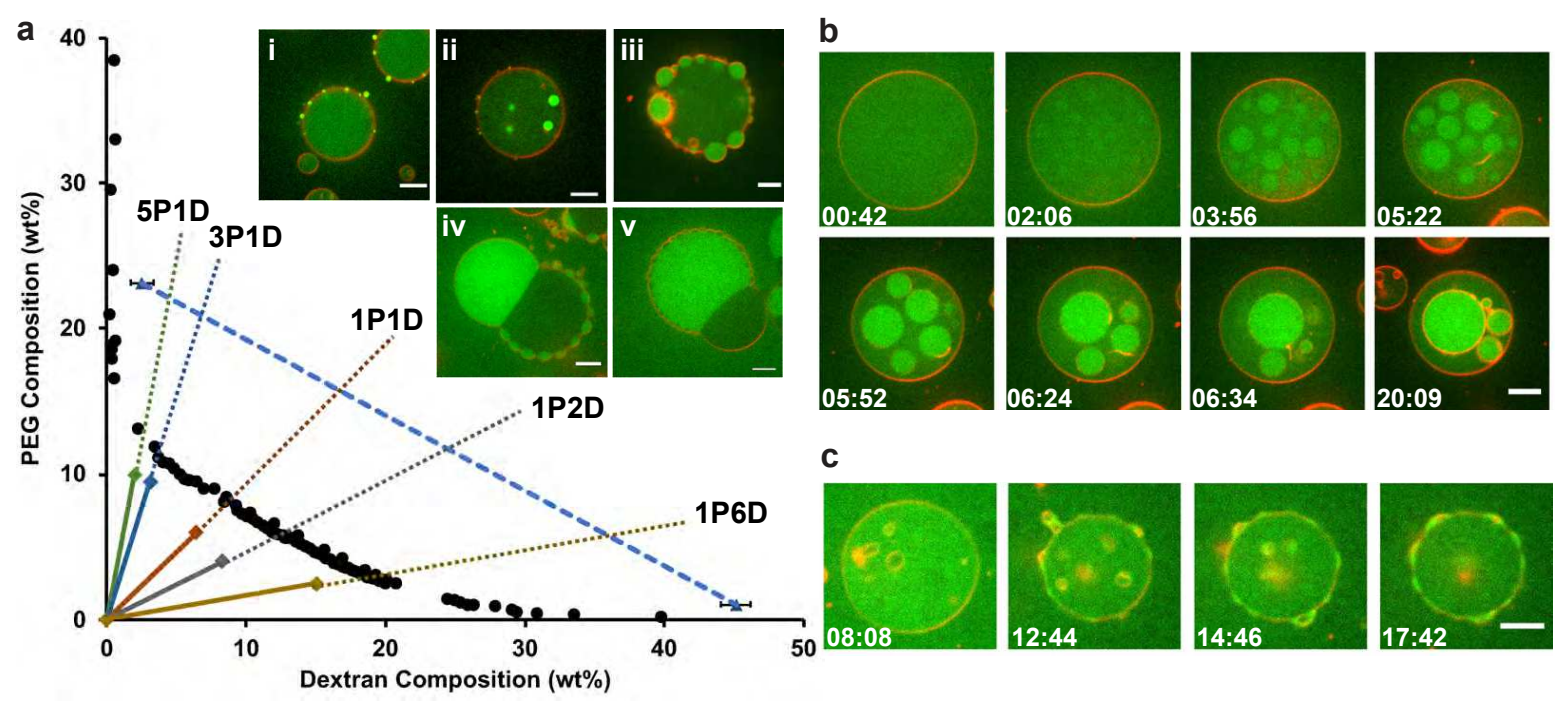

Figure 3. (a) Phase diagram and confocal fluorescence microscopy images of driving LLPS in GUVs with different ratios of PEG $8 \mathrm{~kg} \mathrm{~mol}^{-1}$ and dextran $10 \mathrm{~kg} \mathrm{~mol}^{-1}$. (i-v) confocal fluorescence microscopy image of GUVs encapsulating mixtures of (i) 5:1 (namely 5P1D, Supplementary Video S3), (ii) 3:1 (namely 3P1D, Supplementary Video S4), (iii) 1:1, (iv) 1:2 (namely 1P2D, Supplementary Video S5) and (v) 1:6 (namely 1P6D, Supplementary Video S6) ratios of PEG $8 \mathrm{~kg} \mathrm{~mol}^{-1}$ and dextran $10 \mathrm{~kg} \mathrm{~mol}^{-1}$ doped with $0.001 \%$ AlexaFluor ${ }^{\circledR}$ 488-Dextran upon immersion in (i) 143, (ii) 120, (iii) 143, (iv) 263, and (v) 298mM sucrose solution. All GUVs imaged consist of a mixture containing 96.8\% POPC doped with 2.2\% DOPE-mPEG, 1\% Rho-DOPE. (b) Confocal fluorescence microscopy image of GUVs consists of a mixture containing 96.8\% POPC, 2.2\% DOPE-mPEG, 1\% Rho-DOPE with 4.5\% PEG 8 $\mathrm{kg} \mathrm{mol}{ }^{-1}$ and $2.02 \%$ Dextran $450 \mathrm{~kg} \mathrm{~mol}^{-1}$ subjected to $54 \mathrm{mM}$ sucrose solution (Supplementary Video S7); (c) Confocal fluorescence microscopy image of a single GUV consists of a mixture containing 95\% POPC, 4\% GM1, 1\% Rho-DOPE with $6.0 \%$ PEG $8 \mathrm{~kg}$ $\mathrm{mol}^{-1}$ and $6.4 \%$ Dextran $10 \mathrm{~kg} \mathrm{~mol}^{-1}$ subjected to $143 \mathrm{mM}$ sucrose solution (Supplementary Video S8). All scale bar, $10 \mu \mathrm{m}$. 
a
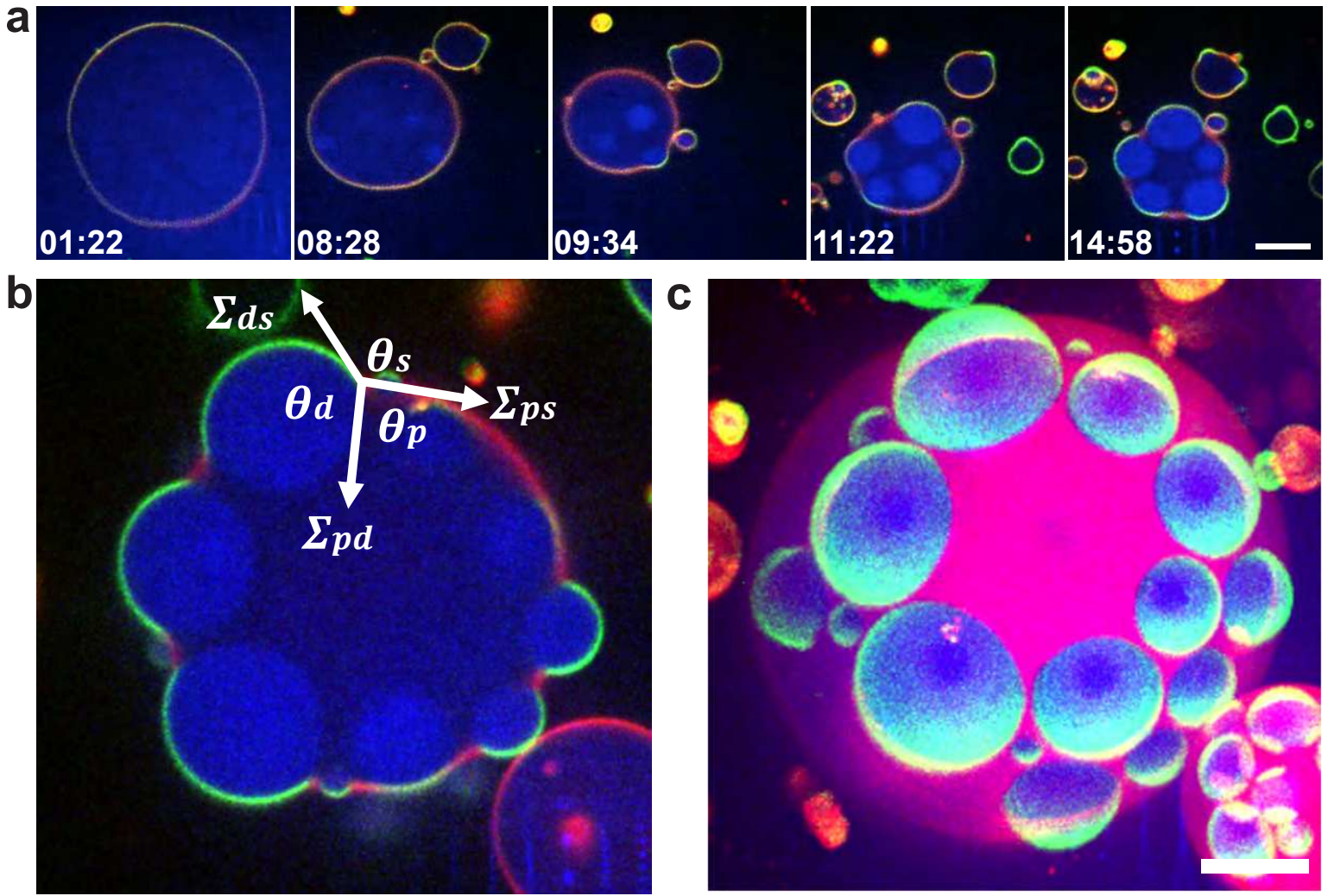

Figure 4. (a) Selected frames, (b) $2 \mathrm{D}$ and (c) 3D images from a time-lapse movie (Supplementary Video S9) confocol fluorescence microscopy images of GUVs encapsulating a mixture of $6.0 \%(\mathrm{w} / \mathrm{w})$ PEG $8 \mathrm{~kg} \mathrm{~mol}^{-1}$ and $6.4 \%(\mathrm{w} / \mathrm{w})$ dextran $10 \mathrm{~kg} \mathrm{~mol}^{-1}$ doped with $0.001 \%$ AlexaFluor ${ }^{\circledR}$ 647-Dextran upon immersion in $157 \mathrm{mM}$ sucrose solution. Three contact angle $\theta_{p}\left(86.72^{\circ}\right), \theta_{d}\left(140.89^{\circ}\right)$, and $\theta_{s}\left(132.38^{\circ}\right)$ corresponding to PEG-rich phase, dextran-rich phase and exterior solution. $\Sigma_{p s}, \Sigma_{p d}$, and $\Sigma_{d s}$ represents three tensions along each contact lines. GUVs imaged consist of a mixture containing POPC: Egg-SM: Cholesterol in a ratio of 2:2:1 doped with 2.2\% DOPE-mPEG, $1 \%$ Rho-DOPE and 3\% NBD-PE. Scale bar, $10 \mu \mathrm{m}$. 
1. Kusumaatmaja, H.; Li, Y.; Dimova, R.; Lipowsky, R., Intrinsic Contact Angle of Aqueous Phases at Membranes and Vesicles. Physical Review Letters 2009, 103 (23), 238103.

2. Hohenberg, P. C.; Halperin, B. I., Theory of dynamic critical phenomena. Reviews of Modern Physics 1977, 49 (3), 435-479. 


\section{Supplementary Files}

This is a list of supplementary files associated with this preprint. Click to download.

- SuATPSSV220210701.avi

- SusuppATPSNatChem20210818.pdf

- SuATPSSV720210701.avi

- SuATPSSV120210701.avi

- SuATPSSV420210701.avi

- SuATPSSV320210701.avi

- SuATPSSV620210701.avi

- SuATPSSV820210701.avi

- SuATPSSV520210701.avi

- SuATPSSV920210701.avi

- SusuppATPSNatChem20210818.pdf 